\title{
An Unusual Case of Rectal Bleeding due to Metastasis from Cholangiocarcinoma
}

\author{
Arcelia Guerson Gil*, Qiang Liu and Sammy Ho \\ Internal Medicine, Montefiore Medical Center, USA
}

ISSN: 2637-7632

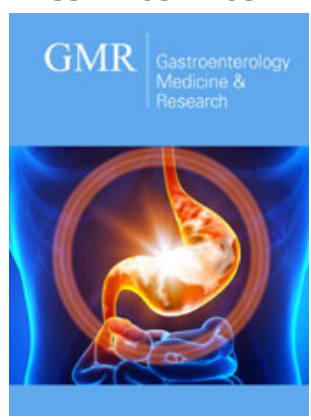

*Corresponding author: Arcelia Guerson Gil, MD, Internal Medicine, Montefiore Medical Center, USA

Submission: 海 July 17, 2020

Published: 制July 27, 2020

Volume 4 - Issue 5

How to cite this article: Arcelia Guerson Gil, Qiang Liu and Sammy Ho. An Unusual Case of Rectal Bleeding due to Metastasis from Cholangiocarcinoma. Gastro Med Res. 4(5). GMR.000599. 2020. DOI: $10.31031 /$ GMR.2020.04.000599

Copyright@ Arcelia Guerson Gil, This article is distributed under the terms of the Creative Commons Attribution 4.0 International License, which permits unrestricted use and redistribution provided that the original author and source are credited.

\section{Introduction}

Cholangiocarcinoma (CCA) is a rare, yet aggressive form of cancer that can originate from the epithelium to anywhere along the bile ducts (BD). Depending on the locations of the CCA, the treatment, prognosis, and diagnosis can be greatly impacted. The primary risk factors are sclerosing cholangitis and choledochal cysts. Anatomically, CCA are classified as intrahepatic (IH) and extrahepatic (EH). EH further divided into distal and perihilar. These subtypes have common features but also important differences that affect the pathogenesis and outcome. Histologically, a great majority of these (90\%) are adenocarcinomas with the remainder being mostly squamous cell carcinomas. It is possible to support a diagnosis of malignant biliary epithelium through immunohistochemical staining. Specifically, cytokeratin-7 (CK-7) positivity is consistent with biliary tract origin. The clinical presentation of CCA varies, with some patients presenting with jaundice. However, most cases are silent and are not diagnosed until the advanced stage. Common sites of metastasis include the liver, peritoneum, and brain. Although rare, colon metastasis has been reported and requires a high index of suspicion for early detection. Here, we present a rare case of a patient with intermittent rectal bleeding secondary to colon metastasis from CCA.

\section{Case Presentation}

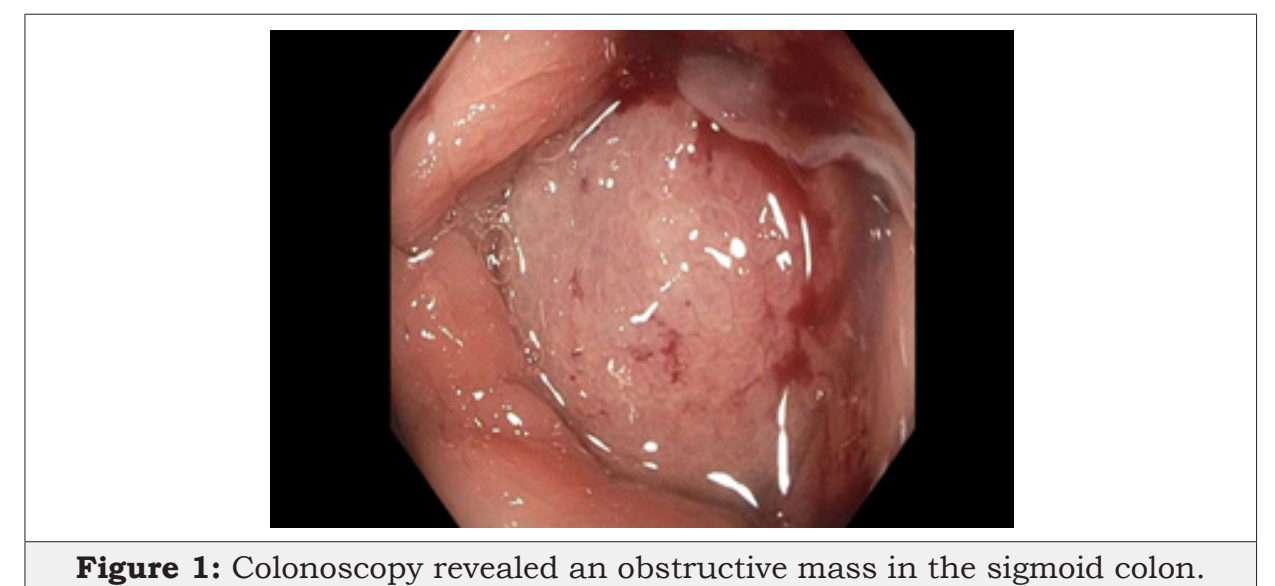

A 49-year-old woman was recently diagnosed with perihilar CCA (Klatskin tumour) after presenting with jaundice, fatigue and early satiety. She underwent multiple surgical procedures including a left lobe hepatectomy, caudate lobe hepatectomy, portal vein resection and reconstruction and a Roux-en-Y hepaticojejunostomy. Subsequently, the patient was started on adjuvant chemotherapy and radiation therapy. Nine months after, the patient presented with a 2-month history of constipation and intermittent rectal bleeding. A colonoscopy was performed for evaluation. An obstructive lesion in the sigmoid was found (Figure 1). Histology 
was consistent with adenocarcinoma; immunohistochemical stains were positive for CK-7 and MUC1 and negative for CK-20 (Figure 2). Morphology of this specimen was similar to previously resected Klatskin tumour. Furthermore, a PET-CT scan revealed increased tracer uptake within the rectosigmoid mass compatible with malignancy. No hypermetabolic nodules were seen in the vicinity of the mass (Figure 3). Therefore, it was determined that this was a colonic metastatic lesion from her primary CCA. Laparoscopic sigmoidoscopy was done and the patient is now on chemotherapy.

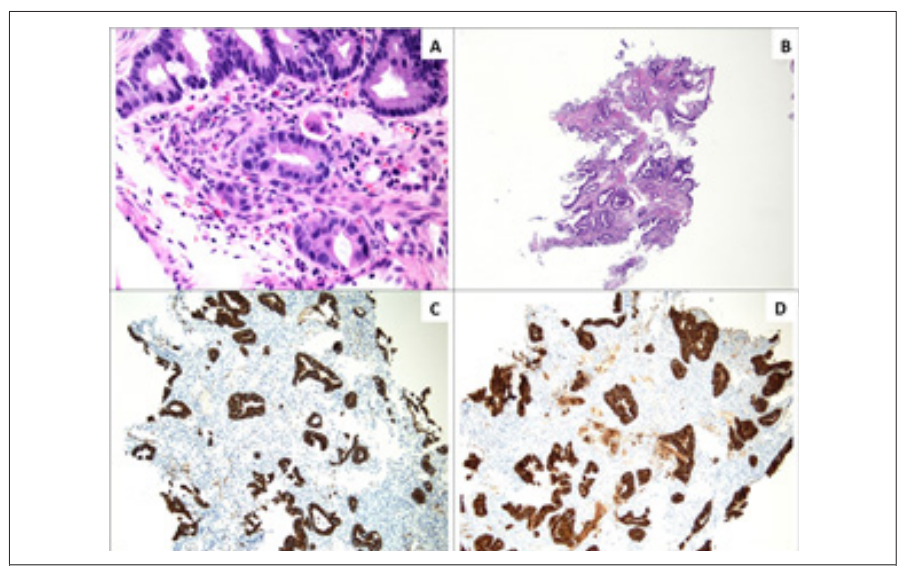

Figure 2: Colonic biopsies.

(A, B): H\&E staining: The adenocarcinoma involves the entire fragment of colonic mucosa with no definitive associated adenomatous epithelium (precursor lesion of colonic adenocarcinoma) seen. Immunohistochemical stains show that the neoplastic cells are positive for CK7. (C): MUC1.

(D): Negative for CK20 and CDX2, the morphology and immunoprofile of the carcinoma, although is not entirely specific, along with known history of cholangiocarcinoma, and absence of other known primary tumors, would support origin from known cholangiocarcinoma.

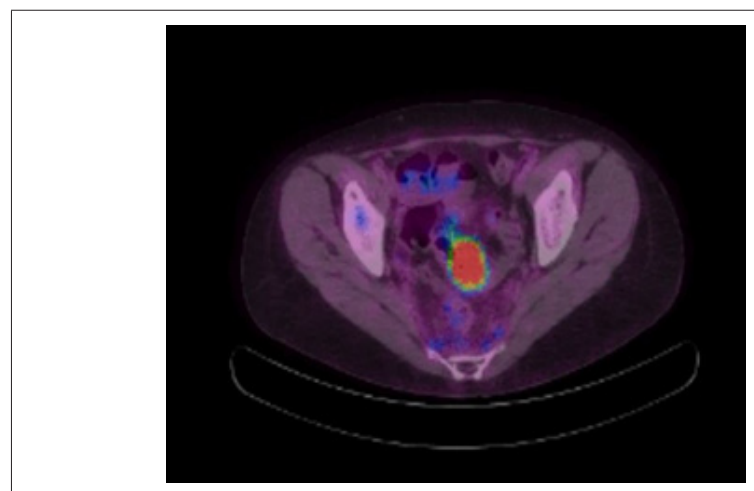

Figure 3: PET-CT scan showed a rectosigmoid mass exhibiting increased activity compatible with malignancy.

\section{Discussion}

CCA spreading to the colon is extremely rare and the reason as to why is poorly understood with only five cases ever reported on this rare anomaly [1]. The most plausible mode of metastasis is hematogenous spread [2,3]. CK-7 and CK-20 immunohistochemical staining is the most reliable way of distinguishing metastatic CCA to the colon from primary colonic adenocarcinoma [4]. In our study, our patient's immunophenotype was CK7+, CK20-, confirming that a metastatic tumour from the CCA, and not a second primary colonic adenocarcinoma. Determining the difference among metastatic colon cancer and primary/secondary colonic cancer is of the paramount importance often impacting prognosis, surgical management, and overall quality of life. Regardless, much remains to be discovered in the pathogenic behaviour of metastatic secondary colorectal cancer. Furthermore, more studies are needed to determine the management approach to these types of metastatic cancers. The question thus remains, do patients with a history of resected CCA benefit from a more frequent screening colonoscopies?

\section{References}

1. Lee YT, Geer DA (1987) Primary liver cancer: Pattern of metastasis. J Surg Oncol 36(1): 26-31.

2. Balthazar EJ, Rosenberg HD, Davidian MM (1979) Primary and metastatic scirrhous carcinoma of the rectum. AJR Am J Roentgenol 132(5): 711-715.

3. Ota H, Azekura K, Seki M (1988) Clinico pathological study on metastatic colorectal cancer. Stomach Intestine 23: 633-643.

4. Rullier A, Le Bail B, Fawaz R, Blanc JF, Saric J, et al. (2000) Cytokeratin 7 and 20 expression in cholangiocarcinoma's varies along the biliary tract but still differs from that in colorectal carcinoma metastasis. Am J Surg Pathol 24(6): 870-876. 